\title{
BDKRB2 +9/-9 Polymorphism Is Associated with Higher Risk for Diabetes Mellitus in the Brazilian General Population
}

\author{
Rafael de Oliveira Alvim, ${ }^{1}$ Paulo C. J. L. Santos, ${ }^{1}$ Raimundo M. Nascimento, ${ }^{2}$ \\ George L. L. M. Coelho, ${ }^{2}$ José G. Mill, ${ }^{3}$ José E. Krieger, ${ }^{1}$ and Alexandre C. Pereira' ${ }^{1}$ \\ ${ }^{1}$ Laboratory of Genetics and Molecular Cardiology, Heart Institute (InCor), University of Sao Paulo Medical School, \\ Avenida Dr. Enéas de Carvalho Aguiar, 44 Cerqueira César, 05403-000 São Paulo, SP, Brazil \\ ${ }^{2}$ Department of Medicine, Ouro Preto Federal University, Ouro Preto, MG, Brazil \\ ${ }^{3}$ Department of Physiology, Espirito Santo Federal University, Vitória, ES, Brazil
}

Correspondence should be addressed to Alexandre C. Pereira, acplbmpereira@gmail.com

Received 3 October 2012; Accepted 1 November 2012

Academic Editor: Aristidis Veves

Copyright ( $) 2012$ Rafael de Oliveira Alvim et al. This is an open access article distributed under the Creative Commons Attribution License, which permits unrestricted use, distribution, and reproduction in any medium, provided the original work is properly cited.

\begin{abstract}
Some mechanisms have been proposed to explain the role of bradykinin on glucose homeostasis and some studies reported that the BDKRB2 +9/-9 polymorphism was associated to the transcriptional activity of the receptor. In this scenario, the main aim of this study was to evaluate the association of the BDKRB2 +9/-9 polymorphism with diabetes mellitus risk in the Brazilian general population. This study included 1,032 subjects of the general urban population. Anthropometrical, blood pressure, biochemical, and genotype analyses for the $B D K R B 2+9 /-9$ bp insertion/deletion polymorphism were performed. Individuals carrying $+9 /+9$ or $+9 /-9$ genotypes had higher glucose values $(84.5 \mathrm{mg} / \mathrm{dL}$ versus $80.6 \mathrm{mg} / \mathrm{dL}$, resp. $)$ and higher frequency of diabetes mellitus ( $7.6 \%$ versus $3.6 \%$, resp.) compared to individuals carrying $-9 /-9$, adjusting for age and gender. In addition, higher diabetes mellitus risk was associated to presence of the $+9 /+9$ or $+9 /-9$ genotypes $(\mathrm{OR}=1.91 ; 95 \% \mathrm{CI}=1.09-4.19 ; P=0.03)$. Our data suggest that the BDKRB2 $+9 /-9$ polymorphism may act as a genetic modulator of glucose homeostasis. It was previously associated to insulin sensitivity, glucose uptake, and insulin secretion, and, in this study, data suggest that the polymorphism may increase susceptibility to chronic metabolic conditions such as diabetes in the Brazilian population.
\end{abstract}

\section{Introduction}

Bradykinin (BK) is a nonapeptide formed by the action of a serine protease called kallikrein. The kallikrein produces kinins which are largely released into interstitial fluid, blood, and glandular tissue, in particular by the pancreas. Several studies have demonstrated the role of $\mathrm{BK}$ in the modulation of important physiological effects such as inflammation, vascular permeability, hypotension, edema, smooth muscle contraction, and glucose homeostasis, and most of these actions are mediated by the $\mathrm{B} 2$ receptor $(\mathrm{B} 2 \mathrm{R})[1,2]$.

Recently, some mechanisms have been proposed to explain the role of BK on glucose homeostasis. Studies have reported that $\mathrm{BK}$ enhances the tyrosine phosphorylation of IRS1 and thus improves the binding affinity of IRS1 with the P85 regulatory subunit of PI3K, which increases the translocation of GLUT4 to the plasma membrane [3]. In addition, the activation of endothelial nitric oxide synthase (eNOS) by BK results in improved blood flow thereby increasing glucose supply to peripheral tissues [4].

$\mathrm{B} 1$ or $\mathrm{B} 2$ receptors mediate the effects of BK. It has been accepted that almost all of the physiologically significant effects of BK, including the metabolic ones, are exerted by activation of the B2R [5]. Based on this information, several studies have focused on insertion/deletion polymorphism in the exon 1 of the BK type 2 receptor gene (BDKRB2). The presence $(+9 \mathrm{bp})$ rather than the absence $(-9 \mathrm{bp})$ of a 9-base pair sequence was associated to lower gene transcriptional activity $[6,7]$. The main aim of this study was to evaluate the association of the BDKRB2 +9/-9 polymorphism with diabetes mellitus risk in the Brazilian general population. 


\section{Methods}

2.1. Study Population. This study included 1,032 subjects of the general urban population selected from the Hearts of Brazil Project (HBP). The design was a transversal and multicenter observational cohort study. The universe of the HBP consisted in the set of inhabitants of Brazilian urban centers with more than 100,000 inhabitants. The HBP sample plan was calculated as 2,500 interviews, distributed in 72 cities from the 5 regions of the country proportionally to the number of inhabitants, per gender and age range, based on data from IBGE (Brazilian Census). In the selected cities, the "households" constituted the second-stage units, with one interview per household. Subjects were separated in self-declared "racial/color" subgroups, according to Brazilian Census, as White, Intermediate (meaning Brown, Pardo in Portuguese), or Black [8-10]. The study protocol was approved by the involved Institutional Ethics Committees and written informed consent was obtained from all participants prior to entering the study.

2.2. Anthropometrical, Blood Pressure, and Biochemical Data. Weight, height, and waist were measured according to a standard protocol. Body mass index (BMI) was calculated and obesity defined as BMI $\geq 30 \mathrm{~kg} / \mathrm{m}^{2}$. Blood pressure was measured in the sitting position with the use of a standard mercury sphygmomanometer on the left arm after 5 minutes' rest. The first and fifth phases of Korotkoff sounds were used for systolic (SBP) and diastolic blood pressure (DBP), respectively. Hypertension was defined as mean SBP $\geq 140 \mathrm{mmHg}$ and/or DBP $\geq 90 \mathrm{mmHg}$ or use of anti-hypertension drugs [11]. Fasting glucose, triglycerides (TG), and total cholesterol (TC) were assayed by technology point-of-care (Roche Diagnostics, Accu-Check). Diabetes was defined as fasting glucose $\geq 126 \mathrm{mg} / \mathrm{dL}$ or hypoglycemic drugs use according to physician prescription (previous diabetes) [12].

2.3. Genotyping. Genomic DNA was extracted from peripheral blood leukocytes following a salting-out method. Genotypes for the BDKRB2 +9/-9 bp insertion/deletion polymorphism was performed by polymerase chain reaction followed by restriction fragment length polymorphism (PCR-RFLP) as previously described [13]. Quality control for these assays was assessed by randomly selecting $10 \%$ samples to be regenotyped by two independent technicians.

2.4. Statistical Analyses. Statistical analyses were carried out using the SPSS 19.0 software (Chicago, IL, USA), with the level of significance set at $P \leq 0.05$. Categorical variables were presented as percentage, whereas continuous variables were presented as mean \pm standard deviation. Differences in the categorical and continuous parameters according to genotypes for the $B D K R B 2$ polymorphism were tested by chi-square test and Student's $t$-test, respectively. Logistic regression analyses were performed to evaluate the association of the BDKRB2 polymorphism with diabetes mellitus plus adjustment for covariates.

\section{Results}

Demographic and clinical data of the studied population samples are shown in Table 1. Genotypic frequencies were $26.8 \%$ for $+9 /+9 ; 53.4 \%$ for $+9 /-9 ; 19.8 \%$ for $-9 /-9$, and frequency of deletion allele was of $47 \%$. Genotypic distribution in the overall sample was in accordance with the Hardy-Weinberg equilibrium $\left(P=0.46, \chi^{2}=0.54\right)$.

Differences in age, gender, race/color, BMI, obesity, abdominal circumference, SBP, DBP, hypertension, TG, and TC according to genotype groups were not observed $(+9 /+9$ plus $+9 /-9$ versus $-9 /-9)$. However, individuals carrying $+9 /+9$ or $+9 /-9$ genotypes had higher glucose values $(84.5 \mathrm{mg} / \mathrm{dL}$ versus $80.6 \mathrm{mg} / \mathrm{dL} ; P=0.04$, resp.) and higher frequency of diabetes mellitus (7.6\% versus $3.6 \%$, resp.) compared to individuals carrying $-9 /-9$, adjusting for age, gender, and BMI (Table 1). In addition, higher diabetes mellitus risk was associated to presence of the $+9 /+9$ or $+9 /-9$ genotypes $(\mathrm{OR}=1.91 ; 95 \% \mathrm{CI}=1.09-4.19 ; P=0.03)($ Table 1$)$.

\section{Discussion}

The main finding of this study was that the BDKRB2 +9/-9 polymorphism is associated with fasting glucose values and with diabetes mellitus risk in the Brazilian population. These genetic associations, although exploratory regarding casual relations, may have a role in the generation of hypothesis to be tested in more controlled studies.

In recent years, numerous studies have demonstrated the important biological role of BK in the modulation of various physiological conditions mediated through its binding to B1 and $\mathrm{B} 2$ receptors $[1,2]$. In addition, a recent study reported that the $\mathrm{B} 2$ receptor mediates the interaction of $\mathrm{BK}$ with pathways related to glucose homeostasis [5].

Some investigations confirmed the key role of BK on insulin sensitivity [14], glucose uptake [15], and insulin secretion by beta cells [16]. Iozzo et al., studying obese and lean individuals, demonstrated that intra-arterial infusion of $\mathrm{BK}$ in the left leg resulted in an increase in glucose uptake restricted to lean subjects [17]. Corroborating the above results, Uehara et al. demonstrated that infusion of BK increased glucose uptake into dog peripheral tissues and that infusion of a BK antagonist abolished this effect on insulin sensitivity [18].

Finally, Yang and Hsu, studying rat pancreas, showed that the infusion of BK increased the insulin release by beta cells. Furthermore, the BK optimized insulin release stimulated by glucose [16]. In this scenario and knowing that the presence (+9bp) rather than the absence ( $-9 \mathrm{bp})$ of a 9-base pair sequence was associated to lower gene transcriptional activity $[6,7]$, our results suggest a role of BK on glucose homeostasis represented by increased risk for diabetes mellitus.

Considering the pivotal role of B2 receptor in the physiological actions of BK, studies evaluating this genetic variant with metabolic phenotypes are still scarce in the literature. Our findings are supported by functional studies in the literature [14-17]. Moreover, previous studies demonstrated the role of the $\mathrm{B} 2$ receptor in the metabolic actions of $\mathrm{BK}$ [5] and association of BDKRB2 +9 allele with lower gene 
TABLE 1: Demographic, clinical, and biochemical data according to genotypes for the BDKRB2 polymorphism.

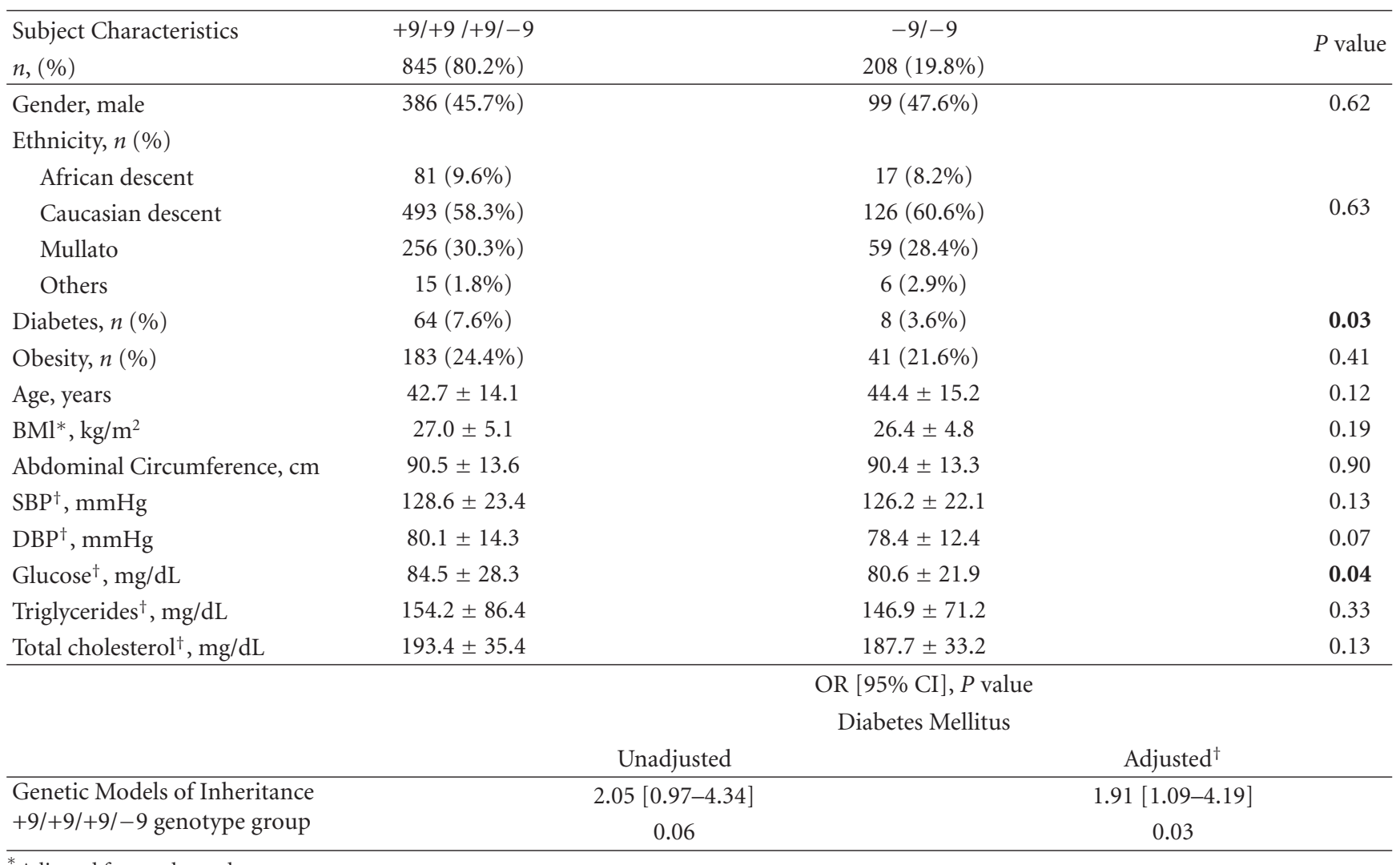

* Adjusted for gender and age.

${ }^{\dagger}$ Adjusted for gender, age, and BMI.

BMI: body mass index; SBP: systolic blood pressure; DBP: diastolic blood pressure.

"Race/color" was classified according to Brazilian Census as White, Intermediate (meaning Brown, Pardo in Portuguese), or Black.

transcriptional activity [7]. Finally, our data may suggest that the reduced transcriptional activity of the B2 receptor presented by +9 allele carriers is associated to lower BK activity, reduced glucose uptake [15], and insulin resistance [14]. Thus, it could generate an unfavorable glucose profile and consequent increased risk for developing diabetes.

There are some limitations in our study. First, we were not able to specifically determine the molecular alteration responsible for the observed association. Nonetheless, in the described scenario, our data plus literature data are well grounded and provide evidence that the BDKRB2 gene may constitute not only a link between biomechanical transduction and diabetes condition, but may also be a fundamental gateway for both epidemiological and mechanistic studies involving diabetes, hypertension, and reninangiotensin system hyperactivity. Second, our examination of glucose has its limitations against the gold standard. However, the classification of the diabetes condition was made according to criteria recommended [12]. Finally, it is not possible to completely exclude the interaction between the use of other genetic markers, ethnicity, concomitant drugs, and other covariates on our findings $[19,20]$.

In conclusion, our data suggest that the BDKRB2 +9/-9 polymorphism may act as a genetic modulator of glucose homeostasis. It was previously associated to insulin sensitivity [14], glucose uptake [15], and insulin secretion by beta cells [16] and, in this study, data suggest that the polymorphism may increase susceptibility to chronic metabolic conditions such as diabetes in the Brazilian population.

\section{Conflict of Interests}

The authors declare that they have no conflict of interests.

\section{Acknowledgments}

P. C. J. L. Santos is recipient of the fellowship from FAPESP, Proc. 2010-17465-8, Brazil. The technical assistance of the Laboratory of Genetics and Molecular Cardiology group, Heart Institute group, and Samaritano Hospital is gratefully acknowledged.

\section{References}

[1] D. Regoli and J. Barabé, "Pharmacology of bradykinin and related kinins," Pharmacological Reviews, vol. 32, no. 1, pp. 146, 1980.

[2] E. S. M. Rocha, W. T. Beraldo, and G. Rosenfeld, "Bradykinin, a hypotensive and smooth muscle stimulating factor released from plasma globulin by snake venoms and by trypsin," American Journal of Physiology, vol. 156, pp. 261-273, 1949. 
[3] T. Shiuchi, T. X. Cui, L. Wu et al., "ACE inhibitor improves insulin resistance in diabetic mouse via bradykinin and NO," Hypertension, vol. 40, no. 3, pp. 329-334, 2002.

[4] H. Beck-Nielsen and L. C. Groop, "Metabolic and genetic characterization of prediabetic states. Sequence of events leading to non-insulin-dependent diabetes mellitus," Journal of Clinical Investigation, vol. 94, no. 5, pp. 1714-1721, 1994.

[5] I. Duka, S. Shenouda, C. Johns, E. Kintsurashvili, I. Gavras, and H. Gavras, "Role of the B2 receptor of bradykinin in insulin sensitivity," Hypertension, vol. 38, no. 6, pp. 1355-1360, 2001.

[6] A. Braun, S. Kammerer, E. Maier, E. Böhme, and A. A. Röscher, "Polymorphisms in the gene for the human B2-bradykinin receptor. New tools in assessing a genetic risk for bradykininassociated diseases," Immunopharmacology, vol. 33, no. 1-3, pp. 32-35, 1996.

[7] C. C. Lung, E. K. L. Chan, and B. L. Zuraw, "Analysis of an exon 1 polymorphism of the $\mathrm{B} 2$ bradykinin receptor gene and its transcript in normal subjects and patients with $\mathrm{C} 1$ inhibitor deficiency," Journal of Allergy and Clinical Immunology, vol. 99, no. 1 I, pp. 134-146, 1997.

[8] M. Makdisse, A. D. C. Pereira, D. D. P. Brasil et al., "Prevalence and risk factors associated with peripheral arterial disease in the hearts of Brazil project," Arquivos Brasileiros de Cardiologia, vol. 91, no. 6, pp. 370-414, 2008.

[9] P. C. J. L. Santos, R. A. G. Soares, D. B. G. Santos et al., "CYP2C19 and ABCB1 gene polymorphisms are differently distributed according to ethnicity in the Brazilian general population," BMC Medical Genetics, vol. 19, no. 12, article 13, 2011.

[10] R. A. G. Soares, P. C. J. L. Santos, G. L. L. MacHado-Coelho et al., "CYP2C9 and VKORC1 polymorphisms are differently distributed in the brazilian population according to selfdeclared ethnicity or genetic ancestry," Genetic Testing and Molecular Biomarkers, vol. 16, no. 8, pp. 957-963, 2012.

[11] "1999 World Health Organization-International Society of Hypertension Guidelines for the Management of Hypertension. Guidelines Subcommittee.," Journal of Hypertension, vol. 17, no. 2, pp. 151-183, 1999.

[12] "Executive summary: standards of medical care in diabetes2011," Diabetes Care, vol. 34, supplement1, pp. S4-S10, 2011.

[13] S. Kammerer, A. Braun, N. Arnold, and A. A. Roscher, "The human bradykinin B2 receptor gene: full length cDNA, genomic organization and identification of the regulatory region," Biochemical and Biophysical Research Communications, vol. 211, no. 1, pp. 226-233, 1995.

[14] E. J. Henriksen, S. Jacob, H. J. Augustin, and G. J. Dietze, "Glucose transport activity in insulin-resistant rat muscle: effects of angiotensin-converting enzyme inhibitors and bradykinin antagonism," Diabetes, vol. 45, no. 1, pp. S125-S128, 1996.

[15] G. Doetze and M. Wicklmayr, "Evidence for a participation of the kallikrein kinin system in the regulation of muscle metabolism during muscular work," FEBS Letters, vol. 74, no. 2, pp. 205-208, 1977.

[16] C. Yang and W. H. Hsu, "Stimulatory effect of bradykinin on insulin release from the perfused rat pancreas," American Journal of Physiology, vol. 268, no. 5, pp. E1027-E1030, 1995.

[17] P. Iozzo, A. Viljanen, M. A. Guzzardi et al., "The interaction of blood flow, insulin, and bradykinin in regulating glucose uptake in lower-body adipose tissue in lean and obese subjects," Journal of Clinical Endocrinology and Metabolism, vol. 97, no. 7, pp. E1192-E1196, 2012.

[18] M. Uehara, H. Kishikawa, S. Isami et al., "Effect on insulin sensitivity of angiotensin converting enzyme inhibitors with or without a sulphydryl group: bradykinin may improve insulin resistance in dogs and humans," Diabetologia, vol. 37, no. 3, pp. 300-307, 1994.

[19] R. O. Alvim, P. C. J. L. Santos, N. E. Ferreira, J. G. Mill, J. E. Krieger, and A. C. Pereira, "Thioredoxin interacting protein (TXNIP) rs7212 polymorphism is associated with arterial stiffness in the Brazilian general population," Journal of Human Hypertension, vol. 26, no. 5, pp. 340-342, 2012.

[20] P. C. J. De Lima Santos, R. De Oliveira Alvim, N. E. Ferreira et al., "Ethnicity and arterial stiffness in Brazil," American Journal of Hypertension, vol. 24, no. 3, pp. 278-284, 2011. 


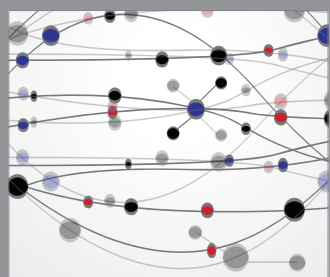

The Scientific World Journal
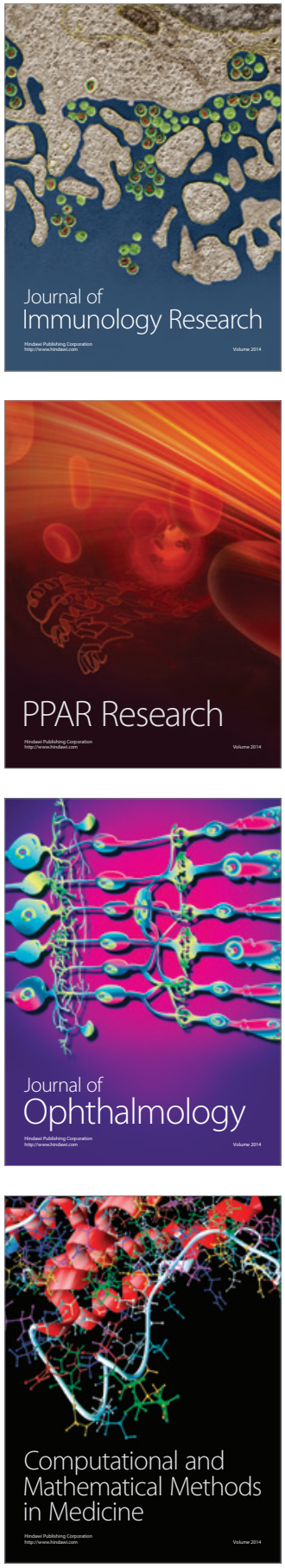

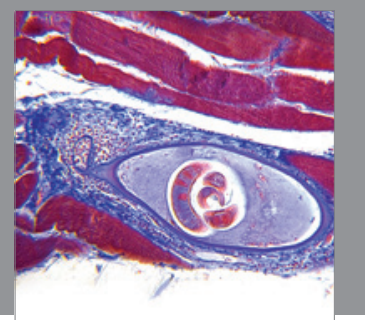

Gastroenterology

Research and Practice
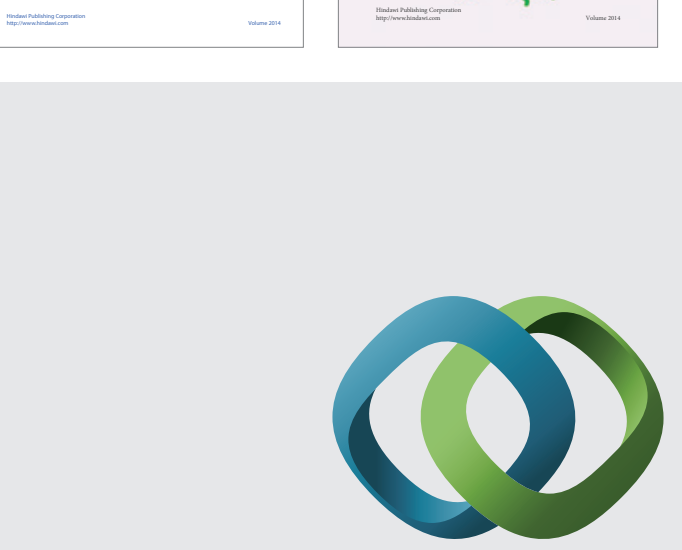

\section{Hindawi}

Submit your manuscripts at

http://www.hindawi.com
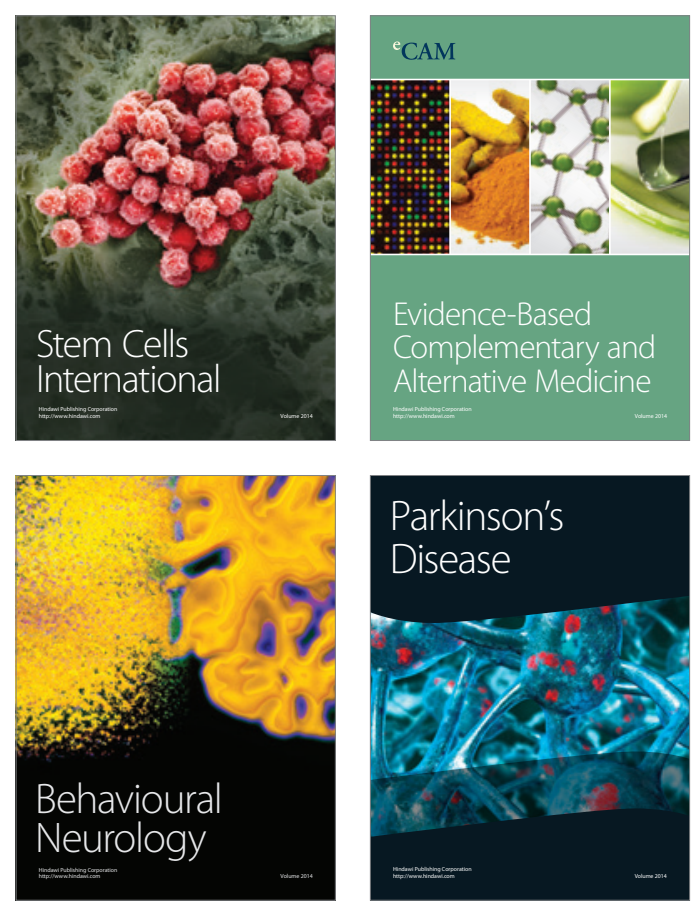

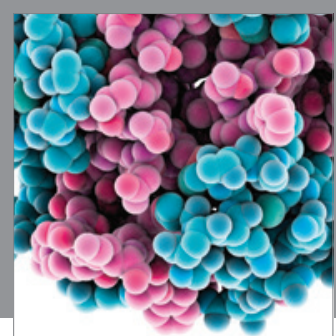

Journal of
Diabetes Research

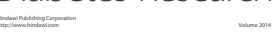

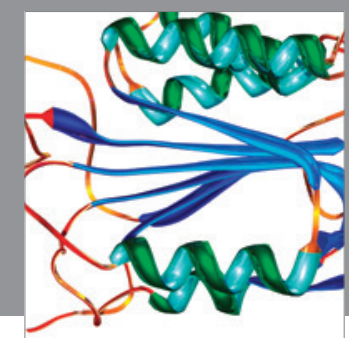

Disease Markers
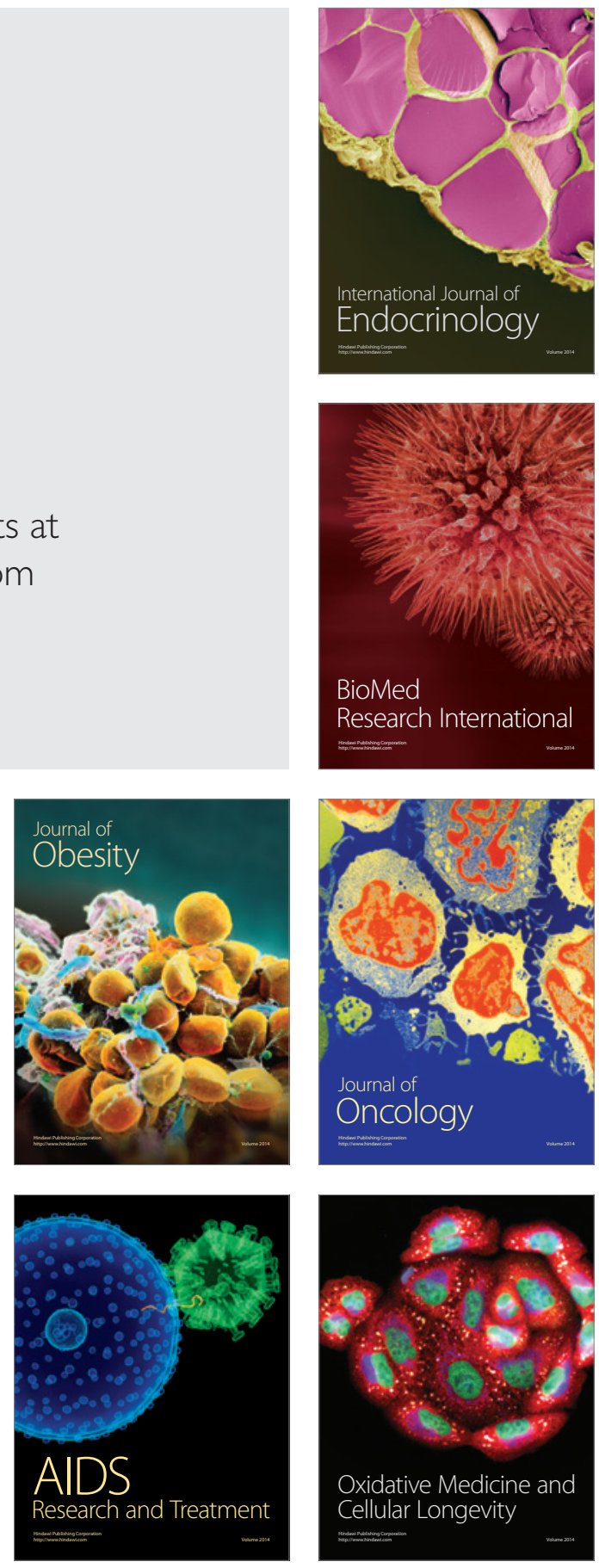